九州大学学術情報リポジトリ

Kyushu University Institutional Repository

\title{
A TRUNCATED PLAY-THE-WINNER PROCEDURE FOR SELECTING THE BEST OF \$ k geqq 3 \$ BINOMIAL POPULATION
}

Schriever, Karl-Heinz

Institut fur Statistik und Mathematische Wirtschaftstheorie Universitat Karlsruhe

https://doi.org/10.5109/13121

出版情報 : 統計数理研究. 18 (1/2), pp. 21-33，1978-03. Research Association of Statistical Sciences

バージョン :

権利関係 : 


\title{
A TRUNCATED PLAY-THE-WINNER PROCEDURE FOR SELECTING THE BEST OF $k \geqq 3$ BINOMIAL POPULATION
}

\author{
By \\ Karl-Heinz SCHRIEVER*
}

(Received August 31, 1977)

\begin{abstract}
Summary
A truncated play-the-winner procedure for selecting the best of $k \geqq 3$ binomial populations is proposed. Sampling always terminates and the maximal number of trials up to a final decision is equal to $k \cdot(r+c-1)$. The large expected sample sizes of the Sobel-Weiss-procedure [4] when the success parameters are small are avoided and the problem of a never ending sampling (using the Sobel-Weiss-procedure) when all success parameters are equal to 0 doesn't occur.
\end{abstract}

\section{Introduction}

The following procedure is a generalization of the Sobel-Berry-procedure [2] for $k \geqq 3$ binomial populations. At the outset it puts the $k$ populations in a random order. Let $A_{1}$ denote the best population, that means the population with the largest success parameter, $A_{2}$ the one following $A_{1}$ in the initial randomization, e.t.c. (continuing in cyclic order) and let $p_{i}$ denote the success parameter of population $A_{i}, i \in\{1,2, \cdots, k\}$. We use play-the-winner sampling with the orderd populations. Sampling terminates whenever one of the $k$ populations yields $r$ successes or all yield $c$ failures (i.e. whenever play-the-winner procedure has gone through $c$ cycles). In either case the population with the larger number of successes is selected. If the number of successes is the same for at least two populations (which can only occur after $c$ cycles), one of these populations is selected randomly. We must determine $r$ and $c$ so that the probability of a correct selection $(P(C S))$ satisfies the following $\left(P^{*} ; \Delta^{*}\right)$-condition :

$$
\begin{gathered}
P(C S) \geqq P^{*} \quad \text { whenever } \quad p_{1}-\max _{i>1} p_{i}=: \Delta \geqq \Delta^{*} \\
\text { with } 1 / k<P^{*}<1 \text { and } \Delta^{*}>0
\end{gathered}
$$

* Institut für Statistik und Mathematische Wirtschaftstheorie Universität Karlsruhe, Federal Republic of Germany. 
The maximal number of trials up to a final decision is equal to $k(r+c-1)$. This may for example occur if $k-1$ of the $k$ populations yield $c$ failures and $r-1$ successes and the $k$-th population yields $c-1$ failures and $r$ successes.

\section{Exact results for $P(C S)$}

Let $F_{i}(r)$ denote the number of $A_{i}$-failures preceding the $r$-th success of $A_{i}$, and let $S_{i}(c)$ denote the number of $A_{i}$-successes preceding the $c$-th failure of $A_{i} ; i \in\{1,2$, $\cdots, k\}$.

The probability of a correct selection is a sum of two terms $N, M$ which are determined by $\left(F_{i}(r)\right)_{i \in\{1, \cdots, k\}}$ and $\left(S_{i}(c)\right)_{i \in\{1, \cdots, k\}}$ respectively. $N$ is the probability of selecting $A_{1}$ before $c$ cycles and $M$ is the probability of selecting $A_{1}$ in exactly $c$ cycles. We obtain:

$$
\begin{aligned}
N & =P\left(F_{1}(r)<F_{2}(r), \cdots, F_{1}(r)<F_{k}(r), 0 \leqq F_{1}(r)<c\right) \\
& +\sum_{n=1}^{k-1} \sum_{\omega \in S_{n}} \sum_{\nu=0}^{k-n-1}\left[\frac { ( k - 1 - n ) ! ( k - \nu - 1 ) ! } { k ! ( k - 1 - n - \nu ) ! } P \left(F_{1}(r)=F_{i_{1}}(r)=\cdots\right.\right. \\
& \left.\left.=F_{i_{n}}(r), F_{1}(r)<F_{j_{1}}(r), \cdots, F_{1}(r)<F_{j_{k-n-1}}(r) ; 0 \leqq F_{1}(r)<c\right)\right]
\end{aligned}
$$

where $S_{n}$ is defined als follows:

$$
\begin{aligned}
S_{n}:= & \left\{\omega=\left(\omega_{1}, \omega_{2}\right) \mid \omega_{1}=\left(i_{1}, \cdots, i_{n}\right), \omega_{2}=\left(j_{1}, \cdots, j_{k-n-1}\right), i_{1}<\cdots<i_{n},\right. \\
& j_{1}<\cdots<j_{k-n-1}, i_{1}, \cdots, i_{n}, j_{1}, \cdots, j_{k-n-1} \in\{2, \cdots, k\} \text { and } \\
& \left.\left\{i_{1}, \cdots, i_{n}\right\} \cap\left\{j_{1}, \cdots, j_{k-n-1}\right\}=\phi\right\} .
\end{aligned}
$$

In the first term of (2.1) the conditional probability of a correct selection is equal to 1 ; in the second term of (2.1) the conditional probability of a correct selection equals the probability that in the initial randomization $A_{1}$ precedes all $A_{l}$ with $l \in\left\{i_{1}, \cdots, i_{n}\right\}$.

The contribution of $\left(S_{i}(c)\right)_{i \in\{1, \cdots, k\}}$ is much simpler to evaluate. We obtain:

$$
\begin{aligned}
M= & P\left(S_{2}(c)<S_{1}(c), \cdots, S_{k}(c)<S_{1}(c), 0<S_{1}(c)<r\right) \\
+ & \sum_{n=1}^{k-1} \sum_{\omega \in S_{n}}\left[\frac { 1 } { n + 1 } P \left(S_{1}(c)=S_{i_{1}}(c)=\cdots=S_{i_{n}}(c),\right.\right. \\
& \left.\left.S_{j_{1}}(c)<S_{1}(c), \cdots, S_{j_{k-n-1}}(c)<S_{1}(c), 0<S_{1}(c)<r\right)\right]
\end{aligned}
$$

The conditional probability of a correct selection equals $1, \frac{1}{n+1}$ in the first and second term of (2.2) respectively.

Using the fact that $F_{i}(r)$ and $S_{i}(c)$ are all negative binomial chance variables, we obtain for $N$, 


$$
\begin{aligned}
N= & \sum_{j=0}^{c-1}\left(\begin{array}{c}
r+j-1 \\
j
\end{array}\right) p_{1}^{r} q_{1}^{j} J_{q_{2}}(j+1, r) \cdots \cdots J_{q_{k}}(j+1, r) \\
+ & \sum_{n=1}^{k-1} \sum_{\omega \in S_{n}} \sum_{\nu=0}^{k-n-1} \sum_{j=0}^{c-1}\left[\frac{(k-1-n) !(k-\nu-1) !}{k !(k-1-n-\nu) !}\left(\begin{array}{c}
r+j-1 \\
j
\end{array}\right) p_{1}^{r} q_{1}^{j} .\right. \\
& \cdot\left(J_{q_{i_{1}}}(j, r)-J_{q_{i_{1}}}(j+1, r)\right) \cdots \cdot\left(J_{q_{i_{n}}}(j, r)-J_{q_{i_{n}}}(j+1, r)\right) \cdot \\
& \left.\cdot J_{q_{j_{1}}}(j+1, r) \cdots \cdot J_{q_{j_{k-n-1}}}(j+1, r)\right],
\end{aligned}
$$

where $J_{q}(\cdot, \cdot)$ denotes an incomplete beta-function.

Let $X_{r, p_{1}}$ denote a negative binomial chance variable with index $r>0$ and success parameter $p_{1}$ and let $E^{c-1}(\gamma)$ denote the expectation of the random variable $\gamma$ truncated at $c-1$, then $N$ may be written as follows:

$$
\begin{aligned}
N= & E^{c-1}\left(\prod_{l=2}^{k} J_{q_{l}}\left(X_{r, p_{1}}+1, r\right)\right)+\sum_{n=1}^{k-1} \sum_{\omega \in S_{n}} \sum_{\nu=0}^{k-n-1}\left[\frac{(k-1-n) !(k-\nu-1) !}{k !(k-1-n-\nu) !} .\right. \\
& \left.\cdot E^{c-1}\left(\prod_{l=1}^{n}\left(J_{q_{i_{l}}}\left(X_{r, p_{1}}, r\right)-J_{q_{i_{l}}}\left(X_{r, p_{1}}+1, r\right)\right) \cdot \prod_{l=1}^{k-n-1} J_{q_{j_{l}}}\left(X_{r, p_{1}}+1, r\right)\right)\right] .
\end{aligned}
$$

In the same way we obtain for $M$ (using (2.2)) :

$$
\begin{aligned}
M & =\sum_{j=0}^{r-1}\left(\begin{array}{c}
c+j+1 \\
j
\end{array}\right) q_{1}^{c} p^{j} J_{q_{2}}(c, j) \cdots \cdots J_{q_{k}}(c, j) \\
& +\sum_{n=1}^{k-1} \sum_{\omega \in S_{n}} \sum_{j=0}^{r-1}\left[\frac{1}{n+1}\left(\begin{array}{c}
c+j+1 \\
j
\end{array}\right) q_{1}^{c} p_{1}^{j}\left(J_{p_{i_{1}}}(j, c)-J_{p_{i_{1}}}(j+1, c)\right) \cdots \cdot\right. \\
& \left.\cdot\left(J_{p_{i_{n}}}(j, c)-J_{p_{i_{n}}}(j+1, c)\right) J_{q_{j_{1}}}(c, j) \cdots \cdots J_{q_{j_{k-n-1}}}(c, j)\right]
\end{aligned}
$$

Using the same notation as above we obtain for $M$ :

$$
\begin{aligned}
M= & E^{r-1}\left(\prod_{l=2}^{k} J_{q_{l}}\left(c, X_{c}, q_{1}\right)\right) \\
& +\sum_{n=1}^{k-1} \sum_{\omega \in S_{n}} \frac{1}{n+1} E^{r-1}\left(\prod_{l=1}^{n}\left(J_{p_{i_{l}}}\left(X_{c, q_{1}}, c\right)-J_{p_{i_{l}}}\left(X_{c, q_{1}}+1, c\right)\right)\right. \\
& \left.\cdot \prod_{l=1}^{k-n-1} J_{q_{j_{l}}}\left(c, X_{c}, q_{1}\right)\right)
\end{aligned}
$$

$P(C S)$ is the sum of (2.4) and (2.6). 


\section{Exact results for $E\left(N_{i}\right)$ and $E(N)$}

Our next step consists in the derivation of the expectations $E\left(N_{i}\right)$; where $N_{i}$ denotes the number of "patients on treatment $i$ "; $i \in\{1,2, \cdots, k\}$. For this purpose we define a random vector $T=\left(T_{1}, \cdots, T_{k}, T_{k+1}\right)$, where $T_{i}$ denotes the number of additional successes needed to declare population $A_{i}$ as best for $i \in\{1, \cdots, k\}$, and $T_{k+1}$ denotes the number of additional failures needed to finish the $c$-th cycle. In a second step we define:

$$
U_{j}^{i}\left(n_{1}, \cdots, n_{k}, f\right):=E\left(N_{i} \mid T=\left(n_{i}, \cdots, n_{k}, f\right), N T=A_{j}\right)
$$

where "NT= $A_{j}$ " means : "the next trial is on $A_{j}$ ".

We obtain the following recursion formulas:

$$
\begin{gathered}
U_{j}^{i}\left(n_{1}, \cdots, n_{k}, f\right)=p_{j} U_{j}^{i}\left(n_{1}, \cdots, n_{j-1}, n_{j}-1, n_{j+1}, \cdots, n_{k}, f\right) \\
+q_{j} U_{j+1}^{i}\left(n_{1}, \cdots, n_{k}, f-1\right)+\delta_{i j}
\end{gathered}
$$

with $\delta_{i j}=1$ for $i=j$ and 0 for $i \neq j ; U_{k+1}^{i}:=U_{1}^{i}$. The boundary conditions for (3.2) are given by:

$$
\begin{aligned}
& U_{j}^{i}\left(n_{1}, \cdots, n_{j-1}, 0, n_{j+1}, \cdots, n_{k}, f\right)=0 \text { for } n_{\nu}>0 \quad \forall \nu \neq j \text { and } f>0 ; \\
& U_{j}^{i}\left(n_{1}, \cdots, n_{k}, 0\right)=0 \text { for } n_{\nu}>0 \quad \forall \nu \in\{1, \cdots, k\}, \quad \forall i, j \in\{1, \cdots, k\}
\end{aligned}
$$

To find a solution of (3.2) satisfying (3.3) we use generating functions $T_{j}^{i}$ defined by

$$
T_{j}^{i}:=T_{j}^{i}\left(x_{1}, \cdots, x_{k}, y\right):=\sum_{n_{1}=1}^{\infty} \cdots \sum_{n_{k}=1}^{\infty} \sum_{f=1}^{\infty} U_{j}^{i}\left(n_{1}, \cdots, n_{k}, f\right) x_{1}^{n_{1}}, \cdots, x_{k}^{n k} y^{f}
$$

Having solved (3.2) we obtain for the expectations:

$$
\begin{aligned}
& E\left(N_{i}\right)=\frac{1}{k} \sum_{j=1}^{k} U_{j}^{i}(r, \cdots, r, k \cdot c), \\
& E(N)=E\left(N_{1}+\cdots+N_{k}\right)=\frac{1}{k} \sum_{i=1}^{k} \sum_{j=1}^{k} U_{j}^{i}(r, \cdots, r, k \cdot c) .
\end{aligned}
$$

Solving (3.2) we have to distinguish two cases; If $j=i$ we obtain:

$$
T_{i}^{i}\left(1-p_{i} x_{i}\right)=q_{i} y T_{i+1}^{i}+\frac{x_{1}}{1-x_{1}} \cdot \cdots \cdot \frac{x_{k}}{1-x_{k}} \cdot \frac{y}{1-y} .
$$

If $j \neq i$ we obtain:

$$
T_{j}^{i}=\frac{q_{j} y}{1-p_{j} x_{j}} T_{j+1}^{i}
$$


Using (3.7) we obtain from (3.6) :

$$
T_{i}^{i}=\frac{1}{D^{*}} \prod_{\substack{\nu=1 \\ \nu \neq i}}^{k}\left(1-p_{\nu} x_{\nu}\right) \frac{y}{1-y} \prod_{\nu=1}^{k} \frac{x_{\nu}}{1-x_{\nu}}
$$

with

$$
D^{*}:=\left(1-p_{1} x_{1}\right) \cdot \cdots \cdot\left(1-p_{k} x_{k}\right)-q_{1} \cdots \cdot q_{k} y^{k}
$$

and from (3.7) follows:

$$
\begin{aligned}
T_{j}^{i}=T_{i}^{i} \prod_{\nu=j}^{i-1} \frac{q_{\nu} y}{1-p_{\nu} x_{\nu}} & \text { if } j<i, \\
T_{j}^{i}=T_{i}^{i} \prod_{\nu=1}^{i-1} \frac{q_{\nu} y}{1-p_{\nu} x_{\nu}} \prod_{\nu=j}^{k} \frac{q_{\nu} y}{1-p_{\nu} x_{\nu}} & \text { if } j>i .
\end{aligned}
$$

We next determine the power series expansions of (3.8), (3.9) and (3.10). The expansion of $\frac{1}{D^{*}}$ is given by:

$$
\begin{aligned}
\frac{1}{D^{*}} & =\sum_{n_{1}=0}^{\infty} \cdots \sum_{n_{k}=0}^{\infty} \sum_{l=0}^{\infty}\left(\begin{array}{c}
n_{1}+l \\
n_{1}
\end{array}\right) \cdots \cdots\left(\begin{array}{c}
n_{k}+l \\
n_{k}
\end{array}\right) p_{1}^{n_{1}} \cdots p_{k}^{n_{k}} q_{1}^{l} \cdots \\
& \cdot q_{k}^{l} x_{1}^{n_{1}} \cdots x_{k}^{n_{k}} \cdot y^{k \cdot l}
\end{aligned}
$$

We have to expand the other factors of the product given in (3.8) and to multiply them with (3.11). The coefficients of the resulting power series are as follows:

$$
U_{i}^{i}\left(\lambda_{1}, \cdots, \lambda_{k}, \lambda_{k+1}\right)=\sum_{l=0}^{\sigma\left(k, \lambda_{k+1}\right)}\left[\frac{1}{q_{i}} J_{q_{i}}\left(l+1, \lambda_{i}\right) \prod_{\substack{\nu=1 \\ \nu \neq i}}^{k} J_{q_{\nu}}\left(l, \lambda_{\nu}\right)\right]
$$

$q_{i} \neq 0$ and $\sigma\left(k, \lambda_{k+1}\right)$ is defined by:

$$
\sigma\left(k, \lambda_{k+1}\right):= \begin{cases}{\left[\frac{\lambda_{k+1}}{k}\right]-1} & \text { if } \operatorname{gcd}\left(k, \lambda_{k+1}\right)=k \\ {\left[\frac{\lambda_{k+1}}{k}\right]} & \text { if } \operatorname{gcd}\left(k, \lambda_{k+1}\right)<k\end{cases}
$$

where $\operatorname{gcd}(a, b)$ denotes the greatest common divisor of $a$ and $b$ and $[a]$ denotes the greatest integral number not greater than $a$. We finally obtain:

$$
U_{i}^{i}(r, \cdots, r, k \cdot c)=\sum_{i=0}^{c-1}\left[\frac{1}{q_{i}} J_{q_{i}}(l+1, r) \prod_{\substack{\nu=1 \\ \nu \neq i}}^{k} J_{q_{\nu}}(l, r)\right]
$$

In the same way we obtain for $j<i,\left(q_{i} \neq 0\right)$ :

$$
U_{j}^{i}\left(\lambda_{1}, \cdots, \lambda_{k}, \lambda_{k+1}\right)=\sum_{l=0}^{\sigma\left(k, \lambda_{k+1}-(i-j)\right)}\left[\prod_{\nu=1}^{j-1} J_{q_{\nu}}\left(l, \lambda_{\nu}\right) \frac{1}{q_{i}} \prod_{\nu=j}^{i} J_{q_{\nu}}\left(l+1, \lambda_{\nu}\right) \prod_{\nu=i+1}^{k} J_{q_{\nu}}\left(l, \lambda_{\nu}\right)\right]
$$




$$
U_{j}^{i}(r, \cdots, r, k \cdot c)=\prod_{l=0}^{c-1}\left[\prod_{\nu=1}^{j-1} J_{q_{\nu}}(l, r) \frac{1}{q_{i}} \prod_{\nu=j}^{i} I_{q_{\nu}}(l+1, r) \prod_{\nu=i+1}^{k} J_{q_{\nu}}(l, r)\right]
$$

and the last expressions of interest are as follows:

$$
\begin{gathered}
j>i, \quad q_{i} \neq 0 \\
U_{j}^{i}\left(\lambda_{1}, \cdots, \lambda_{k}, \lambda_{k+1}\right)=\sum_{l=0}^{\sigma\left(k, \lambda_{k+1}-k+(j-i)\right)}\left[\prod_{\nu=1}^{i} \frac{1}{q_{i}} J_{q_{\nu}}\left(l+1, \lambda_{\nu}\right) \prod_{\nu=i+1}^{j-1} J_{q_{\nu}}\left(l, \lambda_{\nu}\right) \prod_{\nu=j}^{k} J_{q_{\nu}}\left(l+1, \lambda_{\nu}\right)\right], \\
U_{j}^{i}(r, \cdots, r, k \cdot c)=\prod_{l=0}^{c-1}\left[\prod_{\nu=1}^{i} \frac{1}{q_{i}} J_{q_{\nu}}(l+1, r) \prod_{\nu=i+1}^{j-1} J_{q_{\nu}}(l+1, r)\right] .
\end{gathered}
$$

$E\left(N_{i}\right)$ and $E(N)$ are now available from (3.5), (3.14), (3.16) and (3.18).

\section{Asymptotic analysis}

$F_{i}(r)$ is a negative binomial chance variable with index $r$ and success parameter $p_{i} ; i \in\{1, \cdots, k\} . S_{i}(c)$ is a negative binomial chance variable with index $c$ and success parameter $q_{i}, i \in\{1,2, \cdots, k\}$. It follows immediately from their definition that $F_{i}(r)$ and $F_{j}(r)$ are independent for $i \neq j$; the same is true for the random variables $S_{i}(c)$.

We use the following identities :

$$
\begin{aligned}
& F_{\nu}(r)>F_{1}(r) \longleftrightarrow \frac{F_{\nu}(r)-r q_{\nu} / p_{\nu}}{\sqrt{r q_{\nu}} / p_{\nu}}>\frac{p_{\nu}}{p_{1}} \sqrt{\frac{q_{1}}{q_{\nu}}} \frac{F_{1}(r)-r q_{1} / p_{1}}{\sqrt{r q_{1}} / p_{1}}-\frac{\Delta_{1 \nu}}{p_{1}} \sqrt{\frac{r}{q_{\nu}}}, \\
& S_{\nu}(c)<S_{1}(c) \longleftrightarrow \frac{S_{\nu}(c)-c p_{\nu} / q_{\nu}}{\sqrt{c p_{\nu}} / q_{\nu}}<\frac{q_{\nu}}{q_{1}} \sqrt{\frac{p_{1}}{p_{\nu}}} \frac{S_{1}(c)-c p_{1} / q_{1}}{\sqrt{c p_{1}} / q_{1}}+\frac{\Delta_{1 \nu}}{q_{1}} \sqrt{\frac{c}{p_{\nu}}},
\end{aligned}
$$

with

$$
\Delta_{1 \nu}:=p_{1}-p_{\nu} ; \nu \in\{2, \cdots, k\} .
$$

From the central limit theorem follows that for large $r$ and $c$ the random variables

$$
F_{\nu r}:=\frac{F_{\nu}(r)-r q_{\nu} / p_{\nu}}{\sqrt{r q_{\nu}} / p_{\nu}} \quad \text { and } \quad S_{\nu r}:=\frac{S_{\nu}(c)-c p_{\nu} / q_{\nu}}{\sqrt{c p_{\nu}} / q_{\nu}}
$$

may be expressed by standard normal chance variables $X_{\nu}, Y_{\nu}$ respectively. $\left(X_{1}=: X\right.$, $Y_{1}=: Y$ ) Denoting with $V_{\nu r}(\cdot), \mathrm{W}_{\nu r}(\cdot)$ the distribution functions of $F_{\nu r}, S_{\nu r}$ respectively, we obtain from $(2.1)$ :

$$
\begin{aligned}
& P\left(F_{1}(r)<F_{2}(r), \cdots, F_{1}(r)<F_{k}(r), 0 \leqq F_{1}(r)<c\right) \\
& \quad=\prod_{\nu=2}^{k} P\left(\frac{p_{\nu}}{p_{1}} \sqrt{\frac{q_{1}}{q_{\nu}}} F_{1 r}-\frac{\Delta_{1 \nu}}{p_{1}} \sqrt{\frac{r}{q_{\nu}}}<F_{\nu r},-\sqrt{r q_{1}} \leqq F_{1 r} \leqq \frac{c p_{1}-r q_{1}}{\sqrt{r q_{1}}}\right) \\
& =\int_{-\sqrt{r q_{1}}}^{\left(c p_{1}-r q_{1}\right) / \sqrt{q_{1}}} \prod_{\nu=2}^{k}\left(1-V_{\nu r}\left(\frac{p_{\nu}}{p_{1}} \sqrt{\frac{q_{1}}{q_{\nu}}} x-\frac{\Delta_{1 \nu}}{p_{1}} \sqrt{\frac{r}{q_{\nu}}}\right)\right) d V_{1 r}(x) .
\end{aligned}
$$


Using lemma 1 of [4] we obtain :

$$
\int_{-\infty}^{\left(c p_{1}-r q_{1}\right) \sqrt{r q_{1}}} \prod_{\nu=2}^{k}\left(1-\Phi\left(\frac{p_{\nu}}{p_{1}} \sqrt{\frac{q_{1}}{q_{\nu}}} x-\frac{\Delta_{1 \nu}}{p_{1}} \sqrt{\frac{r}{q_{\nu}}}\right)\right) d \Phi(x),
$$

where " $\sim$ " means asymptotic equivalence as $r \rightarrow \infty$. Replacing $x$ by $-x$, we get the simpler version of (4.4), that means:

$$
\int_{\left(r q_{1}-c p_{1}\right) / \sqrt{r q_{1}}}^{\infty} \prod_{\nu=2}^{k} \Phi\left(\frac{p_{\nu}}{p_{1}} \sqrt{\frac{q_{1}}{q_{\nu}}} x+\frac{\Delta_{1 \nu}}{p_{1}} \sqrt{\frac{r}{q_{\nu}}}\right) d \Phi(x),
$$

In the same way we obtain:

$$
\begin{aligned}
& P\left(S_{2}(c)<S_{1}(c), \cdots, S_{k}(c)<S_{1}(c), 0<S_{1}(c)<r\right) \sim \\
& \sim \quad \int_{-\infty}^{\left(r q_{1}-c p_{1}\right) / \sqrt{c p_{1}}} \prod_{\nu=2}^{k} \Phi\left(\frac{q_{\nu}}{q_{1}} \sqrt{\frac{p_{1}}{p_{\nu}}} y+\frac{\Delta_{1 \nu}}{q_{1}} \sqrt{\frac{c}{p_{\nu}}}\right) d \Phi(y) .
\end{aligned}
$$

The second terms of (2.5) and (2.6) tend to 0 using this approximation, and may be neglected. Our first result is therefore:

$$
P(C S) \sim(4.5)+(4.6) .
$$

It follows from the integral expression of the incomplete beta-function $J_{q}(s, t)$, that for fixed $s$ and $t J_{q}(s, t)$ is increasing with $q$. That is why the incomplete betafunctions $J_{q_{\nu}}(\cdot, \cdot)$ in the first term of (2.4) and (2.6) get their smallest value if we make $q_{\nu}$ as small as possible, and that will be the case if we define $p_{\nu}:=p_{2}^{*}$ for all $\nu \in\{2, \cdots, k\} . p_{2}^{*}$ is the second largest success parameter. ( $p_{\mathrm{z}}$ is the parameter of the population $A_{2}$ and will be usually different from $p_{2}^{*}$ ). We obtain:

$$
\begin{aligned}
P(C S) \sim & \int_{\left(r q_{1}-c p_{1}\right) / \sqrt{r q_{1}}}^{\infty}\left(\Phi\left(\frac{p_{2}^{*}}{p_{1}} \sqrt{\frac{q_{1}}{q_{2}^{*}}} x+\frac{\Delta}{p_{1}} \sqrt{\frac{r}{q_{2}^{*}}}\right)\right)^{k-1} d \Phi(x) \\
+ & \int_{-\infty}^{\left(r q_{1}-c p_{1}\right) / \sqrt{c p_{1}}}\left(\Phi\left(\frac{q_{2}^{*}}{q_{1}} \sqrt{\frac{p_{1}}{p_{2}^{*}}} y+\frac{\Delta}{q_{1}} \sqrt{\frac{c}{p_{2}^{*}}}\right)\right)^{k-1} d \Phi(y),
\end{aligned}
$$

with $A:=p_{1}-p_{2}^{*}$. Letting $c \rightarrow \infty$ and holding $r$ fixed, we obtain :

$$
P(C S) \sim \int_{-\infty}^{+\infty}\left(\Phi\left(\frac{p_{2}^{*}}{p_{1}} \sqrt{\frac{q_{1}}{q_{2}^{*}}} x+\frac{\Delta}{p_{1}} \sqrt{\frac{r}{q_{2}^{*}}}\right)\right)^{k-1} d \Phi(x) .
$$

Disregarding the fact, that the random variables

$$
Z_{\nu}:=\frac{p_{2}^{*}}{p_{1}} \sqrt{\frac{q_{1}}{q_{2}^{*}}} X_{1}+\frac{\Delta}{p_{1}} \sqrt{\frac{r}{q_{2}^{*}}}-X_{\nu}
$$


and

$$
Z_{\mu}:=\frac{p_{2}^{*}}{p_{1}} \sqrt{\frac{q_{1}}{q_{2}^{*}}} X_{1}+\frac{\Delta}{p_{1}} \sqrt{\frac{r}{q_{2}^{*}}}-X_{\mu}
$$

are not independent in general, we get the much simpler expression:

$$
P(C S) \sim\left(\Phi\left(\frac{\Delta \sqrt{r}}{\sqrt{p_{1}^{2} q_{2}^{*}+q_{1} p_{2}^{* 2}}}\right)\right)^{k-1} .
$$

From

$$
P(C S)=P^{*} \longleftrightarrow \Phi\left(\frac{\Delta \sqrt{r}}{\sqrt{p_{1}^{2} q_{2}^{*}+q_{1} p_{2}^{* 2}}}\right)=P^{*(1 /(k-1))}
$$

follows that the least favorable configuration is obtained in the same way as in [3], [2], that means :

$$
\min P(C S)=\left(\Phi\left(\Delta^{*} \sqrt{\frac{27 r}{8}}\right)\right)^{k-1}
$$

We obtain from (4.11):

$$
J^{*} \cdot \sqrt{\frac{27 r}{8}}=\Phi^{-1}\left(\sqrt[k-1]{P^{*}}\right)=: \lambda^{*}\left(P^{*}\right)
$$

where $\lambda^{*}\left(P^{*}\right)$ is the $100 P^{* 1 /(k-1)}$-percentile of the standard normal distribution. From (4.12) we obtain the required $r$ value:

$$
r^{*}=r=\frac{8}{27} \cdot\left(\frac{\lambda^{*}\left(P^{*}\right)}{\Delta^{*}}\right)^{2}
$$

In this special case the least favorable configuration is obtained by centering $p_{1}$ and $p_{2}^{*}$ about $2 / 3$.

Letting $r \rightarrow \infty$, holding $c$ fixed and disregarding the fact that $Z_{\nu}$ and $Z_{\mu^{\mu}}$ are not independent in general, we obtain :

$$
P(C S) \sim\left(\Phi\left(\frac{\Delta \sqrt{c}}{\sqrt{q_{1}^{2} p_{2}^{*}+q_{2}^{* 2} p_{1}}}\right)\right)^{k-1}
$$

The argument in (4.10) was minimized by setting $p_{0}:=\frac{1}{2}\left(p_{1}+p_{2}^{*}\right)$. Now we set $q_{0}:=1-p_{0}=\frac{1}{2}\left(q_{1}+q_{2}^{*}\right)$. With fixed $q_{0}$ we obtain in a first step $\Delta=\Delta^{*}$ and then a least favorable configuration $q_{1}$ and $q_{2}^{*}$ centered about $q_{0}=2 / 3$, that means $p_{1}$ and $p_{2}^{*}$ are centered about $p_{0}=1 / 3$. It follows immediately that $\min P(C S)$ is the same as given in (4.11) with $c$ instead of $r$. The required $c$ value is thus:

$$
c^{*}=c=\frac{8}{27} \cdot\left(\frac{\lambda^{*}\left(P^{*}\right)}{\Delta^{*}}\right)^{2}
$$

From (3.14), (3.16) and (3.18) follows that $E\left(N_{i}\right)$ and $E(N)$ are monotone increasing with $r$ and $c$. That is why we conjecture that the best choice among all pairs 
$(r, c)$ satisfying the $\left(P^{*}, \Delta^{*}\right)$-condition (1.1) consists in setting $r=c$. With this we obtain from (4.8):

$$
\begin{aligned}
P(C S) \sim & \int_{\sqrt{r}\left(q_{1}-p_{1}\right) / \sqrt{q_{1}}}^{\infty}\left(\Phi\left(\frac{p_{2}^{*}}{p_{1}} \sqrt{\frac{q_{1}}{q_{2}^{*}}} x+\frac{\Delta}{p_{1}} \sqrt{\frac{r}{q_{2}^{*}}}\right)\right)^{k-1} d \Phi(x) \\
+ & \int_{-\infty}^{\sqrt{r}\left(q_{1}-p_{1}\right) / \sqrt{p_{1}}}\left(\Phi\left(\frac{q_{2}^{*}}{q_{1}} \sqrt{\frac{p_{1}}{p_{2}^{*}}} y+\frac{\Delta}{q_{1}} \sqrt{\frac{r}{p_{2}^{*}}}\right)\right)^{k-1} d \Phi(y) .
\end{aligned}
$$

If $p_{1} \neq q_{1} P(C S)$ is given by (4.10) or (4.14), provided $r$ is large enough. Thus we have only to investigate the special case $p_{1}=q_{1}=\frac{1}{2}$. We obtain:

$$
\begin{aligned}
P(C S) \sim & \int_{0}^{\infty}\left(\Phi\left(p_{2}^{*} \sqrt{\frac{2}{q_{2}^{*}}} x+2 \Delta \sqrt{\frac{r}{q_{2}^{*}}}\right)\right)^{k-1} d \Phi(x) \\
& +\int_{-\infty}^{0}\left(\Phi\left(q_{2}^{*} \sqrt{\frac{2}{p_{2}^{*}}} y+2 \Delta \sqrt{\frac{r}{p_{2}^{*}}}\right)\right)^{k-1} d \Phi(y) .
\end{aligned}
$$

Using the fact that,

$$
q_{2}^{*} \gtrless p_{2}^{*} \longrightarrow q_{2}^{*} \sqrt{\frac{2}{p_{2}^{*}}} x+2 \Delta \sqrt{\frac{r}{p_{2}^{*}}} \gtrless p_{2}^{*} \sqrt{\frac{2}{q_{2}^{*}}} x+2 \Delta \sqrt{\frac{2}{q_{2}^{*}}},
$$

we obtain:

$$
P(C S) \geqq\left\{\begin{array}{l}
\left(\Phi\left(\frac{\Delta \sqrt{r}}{\sqrt{\left(\frac{1}{2}\right)^{2} q_{2}^{*}+\frac{1}{2} p_{2}^{* 2}}}\right)\right)^{k-1} \text { if } q_{2}^{*} \geqq p_{2}^{*}, \\
\left(\Phi\left(\frac{\Delta \sqrt{r}}{\sqrt{\left(\frac{1}{2}\right)^{2} p_{2}^{*}+\frac{1}{2} q_{2}^{* 2}}}\right)\right)^{k-1} \text { if } p_{2}^{*}>q_{2}^{*} .
\end{array}\right.
$$

From (4.10) and (4.14) follow immediately that in both cases $\left(q_{2}^{*} \geqq p_{2}^{*}\right.$ and $\left.p_{2}^{*}>q_{2}^{*}\right)$

$$
\min P(C S) \geqq\left(\Phi\left(\Delta^{*} \sqrt{\frac{27 r}{8}}\right)\right)^{k-1},
$$

and from this we obtain that the pair $\left(r^{*}, r^{*}\right)$, given by (4.13), satisfies the $\left(P^{*}, A^{*}\right)$ condition (1.1). 


\section{Numerical results}

\begin{tabular}{|c|c|c|c|c|c|c|c|c|c|}
\hline \multicolumn{2}{|c|}{$k=3 ; \quad P^{*}=0.90$} & & & & & & & & \\
\hline$p_{1}$ & 0.2 & 0.3 & 0.4 & 0.5 & 0.6 & 0.7 & 0.8 & 0.9 & $r$ \\
\hline$E\left(N \mid \Delta^{*}=0.1\right)$ & 275 & 311 & 358 & 396 & 342 & 281 & 231 & 179 & 79 \\
\hline$E\left(N \mid \Delta^{*}=0.2\right)$ & - & 73 & 82 & 86 & 78 & 64 & 51 & 40 & 20 \\
\hline$E\left(N \mid \Delta^{*}=0.3\right)$ & - & - & 34 & 34 & 32 & 27 & 22 & 17 & 9 \\
\hline$E\left(N \mid \Delta^{*}=0.4\right)$ & - & - & - & 17 & 16 & 14 & 12 & 9 & 5 \\
\hline
\end{tabular}

\begin{tabular}{|c|rrrrrrrrrr}
\hline$k=3 ; \quad P^{*}=0.95$ & & 10.7 & & & & & \\
\hline$p_{1}$ & 0.2 & 0.3 & 0.4 & 0.5 & 0.6 & 0.7 & 0.8 & 0.9 & $r$ \\
\hline$E\left(N \mid \Delta^{*}=0.1\right)$ & 396 & 448 & 516 & 577 & 495 & 408 & 335 & 258 & 114 \\
$E\left(N \mid \Delta^{*}=0.2\right)$ & - & 106 & 120 & 127 & 113 & 93 & 75 & 57 & 29 \\
$E\left(N \mid \Delta^{*}=0.3\right)$ & - & - & 49 & 51 & 46 & 39 & 31 & 24 & 13 \\
$E\left(N \mid \Delta^{*}=0.4\right)$ & - & - & - & 28 & 26 & 23 & 18 & 15 & 8 \\
\hline
\end{tabular}

\begin{tabular}{|c|ccccccccccc|}
\hline$k=3 ; \quad P^{*}=0.99$ & & & & & & & & & \\
\hline$p_{1}$ & 0.2 & 0.3 & 0.4 & 0.5 & 0.6 & 0.7 & 0.8 & 0.9 & $r$ \\
\hline$E\left(N \mid \Delta^{*}=0.1\right)$ & 685 & 774 & 892 & 1010 & 856 & 706 & 578 & 443 & 197 \\
$E\left(N \mid \Delta^{*}=0.2\right)$ & - & 183 & 208 & 225 & 196 & 159 & 128 & 96 & 50 \\
$E\left(N \mid \Delta^{*}=0.3\right)$ & - & - & 85 & 88 & 79 & 65 & 52 & 40 & 22 \\
$E\left(N \mid \Delta^{*}=0.4\right)$ & - & - & - & 47 & 43 & 36 & 29 & 23 & 13 \\
\hline
\end{tabular}

\begin{tabular}{|c|ccccccccccc|}
\hline$k=4 ; \quad P^{*}=0.90$ & & 10.6 & & & & & & \\
\hline$p_{1}$ & 0.2 & 0.3 & 0.4 & 0.5 & 0.6 & 0.7 & 0.8 & 0.9 & $r$ \\
\hline$E\left(N \mid \Delta^{*}=0.1\right)$ & 450 & 508 & 584 & 649 & 555 & 454 & 369 & 278 & 98 \\
$E\left(N \mid \Delta^{*}=0.2\right)$ & - & 120 & 134 & 141 & 125 & 101 & 80 & 60 & 25 \\
$E\left(N \mid \Delta^{*}=0.3\right)$ & - & - & 53 & 54 & 49 & 41 & 32 & 24 & 11 \\
$E\left(N \mid \Delta^{*}=0.4\right)$ & - & - & - & 31 & 29 & 24 & 20 & 15 & 7 \\
\hline
\end{tabular}




\begin{tabular}{|c|rrrrrrrrrrr|}
\hline$k=4 ; \quad P^{*}=0.95$ & & & 10.4 & & & & & & & \\
\hline$p_{1}$ & 0.2 & 0.3 & 0.4 & 0.5 & 0.6 & 0.7 & 0.8 & 0.9 & $r$ \\
\hline$E\left(N \mid \Delta^{*}=0.1\right)$ & 615 & 694 & 798 & 894 & 761 & 624 & 506 & 379 & 134 \\
$E\left(N \mid \Delta^{*}=0.2\right)$ & - & 162 & 184 & 195 & 171 & 138 & 109 & 80 & 34 \\
$E\left(N \mid \Delta^{*}=0.3\right)$ & - & - & 73 & 75 & 68 & 56 & 44 & 33 & 15 \\
$E\left(N \mid \Delta^{*}=0.4\right)$ & - & - & - & 40 & 37 & 31 & 25 & 19 & 9 \\
\hline
\end{tabular}

\begin{tabular}{|c|c|c|c|c|c|c|c|c|c|}
\hline$k=4 ; \quad P^{*}=$ & & & & & & & & & \\
\hline$p_{1}$ & 0.2 & 0.3 & 0.4 & 0.5 & 0.6 & 0.7 & 0.8 & 0.9 & $r$ \\
\hline$E\left(N \mid \Delta^{*}=0.1\right)$ & 1000 & 1129 & 1298 & 1469 & 1238 & 1016 & 823 & 613 & 218 \\
\hline$E\left(N \mid \Delta^{*}=0.2\right)$ & 一 & 262 & 298 & 321 & 277 & 223 & 176 & 128 & 55 \\
\hline$E\left(N \mid \Delta^{*}=0.3\right)$ & - & - & 124 & 129 & 114 & 92 & 72 & 53 & 25 \\
\hline$E\left(N \mid \Delta^{*}=0.4\right)$ & - & - & - & 65 & 58 & 48 & 38 & 28 & 14 \\
\hline
\end{tabular}

\begin{tabular}{|c|rrrrrrrrrr|}
\hline$k=5 ; \quad P^{*}=0.90$ & & & 1 & & & & & & \\
\hline$p_{1}$ & 0.2 & 0.3 & 0.4 & 0.5 & 0.6 & 0.7 & 0.8 & 0.9 & $r$ \\
\hline$E\left(N \mid \Delta^{*}=0.1\right)$ & 638 & 720 & 827 & 921 & 783 & 640 & 515 & 381 & 112 \\
$E\left(N \mid \Delta^{*}=0.2\right)$ & - & 165 & 186 & 195 & 172 & 138 & 108 & 78 & 28 \\
$E\left(N \mid \Delta^{*}=0.3\right)$ & - & - & 77 & 79 & 71 & 58 & 45 & 33 & 13 \\
$E\left(N \mid \Delta^{*}=0.4\right)$ & - & - & - & 37 & 34 & 29 & 23 & 17 & 7 \\
\hline
\end{tabular}

\begin{tabular}{|c|rrrrrrrrrr}
\hline$k=5 ; \quad P^{*}=0.95$ & & \multicolumn{1}{c|}{} & \multicolumn{1}{c|}{} & & & & & \\
\hline$p_{1}$ & 0.2 & 0.3 & 0.4 & 0.5 & 0.6 & 0.7 & 0.8 & 0.9 & $r$ \\
\hline$E\left(N \mid \Delta^{*}=0.1\right)$ & 843 & 952 & 1093 & 1225 & 1038 & 848 & 683 & 503 & 148 \\
$E\left(N \mid \Delta^{*}=0.2\right)$ & - & 218 & 246 & 261 & 228 & 183 & 143 & 102 & 37 \\
$E\left(N \mid \Delta^{*}=0.3\right)$ & - & - & 102 & 105 & 94 & 76 & 59 & 43 & 17 \\
$E\left(N \mid \Delta^{*}=0.4\right)$ & - & - & - & 55 & 50 & 41 & 32 & 24 & 10 \\
\hline
\end{tabular}




\begin{tabular}{|c|c|c|c|c|c|c|c|c|c|}
\hline \multicolumn{2}{|c|}{$k=5 ; \quad P^{*}=0.99$} & & & & & & & & \\
\hline$p_{1}$ & 0.2 & 0.3 & 0.4 & 0.5 & 0.6 & 0.7 & 0.8 & 0.9 & $r$ \\
\hline$E\left(N \mid \Delta^{*}=0.1\right)$ & 1333 & 1505 & 1728 & 1955 & 1642 & 1342 & 1079 & 790 & 234 \\
\hline$E\left(N \mid J^{*}=0.2\right)$ & - & 347 & 393 & 424 & 364 & 291 & 226 & 160 & 59 \\
\hline$E\left(N \mid \Delta^{*}=0.3\right)$ & - & - & 158 & 164 & 144 & 115 & 89 & 63 & 26 \\
\hline$E\left(N \mid \Delta^{*}=0.4\right)$ & - & - & - & 84 & 75 & 61 & 47 & 34 & 15 \\
\hline
\end{tabular}

\begin{tabular}{|c|rrrrrrrrrr|}
\hline$k=6 ; \quad P^{*}=0.90$ & & & & & & & & & & \\
\hline$p_{1}$ & 0.2 & 0.3 & 0.4 & 0.5 & 0.6 & 0.7 & 0.8 & 0.9 & $r$ \\
\hline$E\left(N \mid \Delta^{*}=0.1\right)$ & 838 & 945 & 1084 & 1209 & 1025 & 834 & 669 & 488 & 123 \\
$E\left(N \mid \Delta^{*}=0.2\right)$ & - & 217 & 214 & 257 & 225 & 180 & 139 & 99 & 31 \\
$E\left(N \mid \Delta^{*}=0.3\right)$ & - & - & 99 & 100 & 90 & 73 & 56 & 40 & 14 \\
$E\left(N \mid \Delta^{*}=0.4\right)$ & - & - & - & 50 & 46 & 39 & 30 & 22 & 8 \\
\hline
\end{tabular}

\begin{tabular}{|c|rrrrrrrrrr|}
\hline$k=6 ; \quad P^{*}=0.95$ & & & & & & & & & \\
\hline$p_{1}$ & & & & & & & & & \\
\hline & 0.2 & 0.3 & 0.4 & 0.5 & 0.6 & 0.7 & 0.8 & 0.9 & $r$ \\
\hline$E\left(N \mid \Delta^{*}=0.1\right)$ & 1089 & 1229 & 1410 & 1582 & 1336 & 1089 & 872 & 634 & 160 \\
$E\left(N \mid \Delta^{*}=0.2\right)$ & - & 280 & 316 & 335 & 292 & 232 & 180 & 126 & 40 \\
$E\left(N \mid \Delta^{*}=0.3\right)$ & - & - & 128 & 131 & 117 & 94 & 72 & 51 & 18 \\
$E\left(N \mid \Delta^{*}=0.4\right)$ & - & - & - & 64 & 58 & 48 & 37 & 27 & 10 \\
\hline
\end{tabular}

The expected sample size $E(N)$ is increasing with $k . E(N)$ is already greater than 1000 for $k=3, P^{*}=0.99, p_{1}=0.5$ and $\Delta^{*}=0.1$. $E(N)$ is relatively small, even for large $k$, if the difference between the success parameters of the best and second best population is significant, that means greater than 0.4 .

\section{Acknowledgement:}

The author would like to thank H. Martin for computing the tables of section 5 . 


\section{References}

[1] Asaio, Сh., Jojima, K. and Sugimura, M., Extended versions of sequential optimum selection plan with Play-the-Winner sampling and the stopping iules in a finite population, Proceedings in Computational Statistics, 1976 (W. Berlin), Physica-Verlag, 277-284.

[2] BerRy, D.A. and SOBel, M., An improved procedure for selecting the better of two Bernoulli populations, Jasa, 68 (1973), 979-984.

[3] Schriever, K.-H., A Play-the-Winner Procedure for selecting the best of $k \geqq 3$ binomial populations. Preprint series of the Institute of Statistics and Mathematical Economics University of Karlsruhe, Federal Republic of Germany, No. 23, May 1977.

[4] Sobel, M. and Weiss, G.H., Play-the-Winner rule and inverse sampling for selecting the best of $k \geqq 3$ binomial populations. Ann. Math. Statist., 48 (1972), 1808-1826.

[5] Sobel, M. and WeIss, G.H., Play-the-Winner rule and inverse sampling in selecting the better of two binomial populations. Jasa, 66 (1971), 545-551. 